\title{
Monotone iterative method for a $p$-Laplacian boundary value problem with fractional conformable derivatives
}

\author{
Jianfang Qin' ${ }^{1}$ Guotao Wang ${ }^{1,2}$, Lihong Zhang ${ }^{1 *}$ (D) and Bashir Ahmad ${ }^{2}$
}

"Correspondence:

zhanglih149@126.com

'School of Mathematics and

Computer Science, Shanxi Normal

University, Linfen, People's Republic

of China

Full list of author information is

available at the end of the article

\begin{abstract}
By using monotone iterative method, the extremal solutions and the unique solution are obtained for a nonlinear fractional $p$-Laplacian boundary value problem involving fractional conformable derivatives and nonlocal integral boundary conditions. Comparison theorems related to the proposed study are also proved. The paper concludes with an illustrative example for the main result.
\end{abstract}

Keywords: Fractional conformable derivative; $p$-Laplacian operator; Nonlocal integral boundary condition; Extremal solution; Monotone iterative method

\section{Introduction}

Fractional calculus provides powerful tools to deal with complex phenomena occurring in various areas of applied and technical sciences such as control theory, optical and thermal systems, rheology, materials and mechanical systems, robotics, etc. Numerous researchers have investigated different aspects (existence, uniqueness, stability, etc.) of fractional differential equations involving Caputo, Riemann-Liouville, Hadamard type derivatives, for instance, see [1-10]. For some recent results on Riemann-Liouville fractional differential equations, we refer the reader to the articles [11-15] and the references cited therein. Fractional $p$-Laplacian boundary value problems also received considerable attention, for example, see [16-26]. The literature on fractional differential equations equipped with integral boundary conditions also contains a variety of interesting results [27-32].

Monotone iterative method is found to be an important and efficient method to obtain sequences of monotone solutions for initial and boundary value problems. For some applications of this technique to nonlinear fractional differential equations, see [15, 3343]. In 2017, Jarad et. al. [44] proposed a new fractional derivative, which is known as fractional conformable derivative (see definition (2.4)). To the best of the authors' knowledge, the fractional $p$-Laplacian problem involving fractional conformable derivatives is yet to be investigated. In this paper, we apply monotone iterative method to prove the existence of extremal and uniqueness of solutions for the following nonlinear fractional $p$-Laplacian problem involving fractional conformable derivatives and nonlocal integral

(c) The Author(s) 2019. This article is distributed under the terms of the Creative Commons Attribution 4.0 International License (http://creativecommons.org/licenses/by/4.0/), which permits unrestricted use, distribution, and reproduction in any medium, provided you give appropriate credit to the original author(s) and the source, provide a link to the Creative Commons license, and indicate if changes were made. 
boundary condition:

$$
\left\{\begin{array}{l}
{ }_{0}^{\beta} D^{\alpha}\left(\phi_{p}\left({ }_{0}^{\gamma} D^{\alpha} h(t)\right)\right)=f\left(t, h(t),{ }_{0}^{\gamma} D^{\alpha} h(t)\right), \quad t \in(0, d], d>0, \\
\left.t^{\frac{\alpha(1-\beta)}{p-1} \gamma}{ }_{0}^{\alpha} h(t)\right|_{t=0}=\int_{0}^{\tau} a(s) h(s) d s, \quad g(\tilde{h}(0), \tilde{h}(d))=0, \tau \in(0, d),
\end{array}\right.
$$

where $0<\alpha, \gamma, \beta \leq 1, \phi_{p}(t)=|t|^{p-2} t, a \in C([0, d],[0, \infty)), f \in C\left([0, d] \times \mathbb{R}^{2}, \mathbb{R}\right), g \in$ $C\left(\times \mathbb{R}^{2}, \mathbb{R}\right), \phi_{p}, p>1$, denotes the $p$-Laplacian operator and $\phi_{p}^{-1}=\phi_{q}, \frac{1}{p}+\frac{1}{q}=1, \tilde{h}(0)=$ $\left.t^{\alpha(1-\gamma)} h(t)\right|_{t=0}, \tilde{h}(d)=\left.t^{\alpha(1-\gamma)} h(t)\right|_{t=d}$, and ${ }_{0}^{\gamma} D^{\alpha}$ is the fractional conformable derivative of order $\gamma$.

We emphasize that the results obtained for problem (1.1) are new and significantly contribute to the existing literature on $p$-Laplacian problems with fractional conformable derivatives. In order to establish the desired results, we prove two comparison theorems related to the problem at hand, which are presented in Sect. 2 . The main results are presented in Sect. 3.

\section{Preliminaries and lemmas}

For $\alpha, \gamma \in(0,1)$, we denote by $C_{\alpha(1-\gamma)}([0, d], \mathbb{R})$ a Banach space

$$
\left\{h \in C((0, d], \mathbb{R}): t^{\alpha(1-\gamma)} h \in C([0, d], \mathbb{R})\right\}
$$

endowed with the norm $\|h\|_{C_{\alpha(1-\gamma)}}=\sup _{t \in[0, d]} t^{\alpha(1-\gamma)}|h(t)|$.

Let

$$
Y=\left\{h(t) \in C_{\alpha(1-\gamma)}([0, d], \mathbb{R}):{ }_{0}^{\gamma} D^{\alpha} h(t) \in C_{k}([0, d], \mathbb{R}) \text { and }\left.t_{0}^{k \gamma} D^{\alpha} h(t)\right|_{t=0}=\varepsilon\right\},
$$

where $0<\alpha, \gamma<1, k=\frac{\alpha(1-\beta)}{p-1}, \varepsilon=\int_{0}^{\tau} a(s) h(s) d s$, be a Banach space equipped with the norm $\|h\|_{Y}=\max \left\{\sup _{t \in[0, d]} t^{\alpha(1-\gamma)}|h(t)|,\left.\sup _{t \in[0, d]}\right|_{0} ^{\gamma} D^{\alpha} h(t) \mid\right\}$.

Definition 2.1 ([44]) The Riemann-Liouville type fractional conformable integral of order $\gamma \in \mathbb{C}, \operatorname{Re}(\gamma) \geq 0$ is defined by

$$
{ }_{a}^{\gamma} I^{\alpha} h(t)=\frac{1}{\Gamma(\gamma)} \int_{a}^{t}\left(\frac{(t-a)^{\alpha}-(s-a)^{\alpha}}{\alpha}\right)^{\gamma-1} h(s) \frac{d s}{(s-a)^{1-\alpha}} .
$$

Definition 2.2 ([44]) The fractional conformable derivative of Riemann-Liouville type of order $\gamma \in \mathbb{C}, \operatorname{Re}(\gamma) \geq 0$ is defined by

$$
\begin{aligned}
{ }_{a}^{\gamma} D^{\alpha} h(t) & ={ }_{a}^{n} \mathcal{T}^{\alpha}\left(\begin{array}{c}
n-\gamma \\
a
\end{array} I^{\alpha}\right) h(t) \\
& =\frac{a}{\Gamma(n-\gamma)} \int_{a}^{t}\left(\frac{(t-a)^{\alpha}-(s-a)^{\alpha}}{\alpha}\right)^{n-\gamma-1} h(s) \frac{d s}{(s-a)^{1-\alpha}},
\end{aligned}
$$

where

$$
n=[\operatorname{Re}(\gamma)]+1, \quad{ }_{a}^{n} \mathcal{T}^{\alpha}=\underbrace{{ }_{a} \mathcal{T}^{\alpha}{ }_{a} \mathcal{T}^{\alpha} \cdots{ }_{a} \mathcal{T}^{\alpha}}_{n \text { times }}
$$


and ${ }_{a} \mathcal{T}^{\alpha}$ is the conformable differential operator [45]

$$
{ }_{a} \mathcal{T}^{\alpha} h(t)=(t-a)^{1-\alpha} h^{\prime}(t)
$$

Lemma $2.1([44])$ Let $0<\operatorname{Re}(\gamma)<1, n=-[-\operatorname{Re}(\gamma)], f \in L((0, d), \mathbb{R})$. Then

$$
{ }_{a}^{\gamma} I^{\alpha}\left({ }_{a}^{\gamma} D^{\alpha} h(t)\right)=h(t)-\frac{{ }_{a}^{\gamma-1} D^{\alpha} h(a)}{\alpha^{\gamma-1} \Gamma(\gamma)}(t-a)^{\alpha \gamma-\alpha} .
$$

Let us first consider the problem

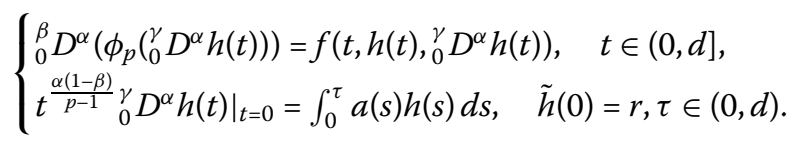

Applying Lemma 2.1 to problem (2.7) with $l(t)=\phi_{p}\left({ }_{0}^{\gamma} D^{\alpha} h(t)\right)$ and $\tilde{h}(0)=r$, we obtain

$$
h(t)=r t^{\alpha(\gamma-1)}+\frac{1}{\Gamma(\gamma)} \int_{0}^{t}\left(\frac{t^{\alpha}-s^{\alpha}}{\alpha}\right)^{\gamma-1} \phi_{q}(l(s)) \frac{d s}{s^{1-\alpha}}=: B l(t)
$$

and

$$
\phi_{p}\left(t^{\frac{\alpha(1-\beta)}{p-1}}{ }_{0} D^{\alpha} h(t)\right)=t^{\alpha(1-\beta)} \phi_{p}\left({ }_{0}^{\gamma} D^{\alpha} h(t)\right)=t^{\alpha(1-\beta)} l(t) .
$$

Thus problem (2.7) takes the form

$$
\left\{\begin{array}{l}
{ }_{0}^{\beta} D^{\alpha} l(t)=f\left(t, B l(t), \phi_{q}(l(t))\right), \quad t \in(0, d], \\
\left.t^{\alpha(1-\beta)} l(t)\right|_{t=0}=\phi_{p}\left[\int_{0}^{\tau} a(s) h(s) d s\right], \quad \tau \in(0, d) .
\end{array}\right.
$$

If (2.10) has a solution $l(t)$, then we get a solution $h(t)$ of Eq. (2.7) after inserting $l(t)$ in Eq.(2.8). This shows the existence of a solution for problem (2.10).

In the following lemma, we use $\|h\|_{*}=\sup _{t \in[0, d]}|h(t)|$.

Lemma 2.2 Suppose that $f \in C\left([0, d] \times \mathbb{R}^{2}, \mathbb{R}\right), 0<\alpha, \beta<1$, and there exists a nonnegative bounded integrable function $M$ on $[0, d]$ such that

$$
\left|f\left(t, h_{1}, h_{2}\right)-f\left(t, l_{1}, l_{2}\right)\right| \leq M(t)\left|\phi_{p}\left(l_{2}\right)-\phi_{p}\left(h_{2}\right)\right|, \quad t \in(0, d] .
$$

Then problem (2.10) has a unique solution $l(t) \in C_{\alpha(1-\beta)}([0, d], \mathbb{R})$, if

$$
\frac{d^{\alpha(\beta-1)} \xi^{p-2} \eta^{q-2}}{\Gamma(\gamma+1)} \int_{0}^{\tau} a(s)\left(\frac{s^{\alpha}}{\alpha}\right)^{\gamma-1} d s+\frac{M d^{\alpha \beta}}{\Gamma(\beta+1) \alpha^{\beta}}<1,
$$

where $\xi$ takes the values between $\int_{0}^{\tau} a(s)_{0}^{\gamma} I^{\alpha} \phi_{q}(h(s)) d s$ and $\int_{0}^{\tau} a(s)_{0}^{\gamma} I^{\alpha} \phi_{q}(l(s)) d s$, the values of $\eta$ remain between $h(u)$ and $l(u)$, and $M=\sup _{t \in[0, d]}|M(t)|$. 
Win et al. Boundary Value Problems

(2019) 2019:145

Page 4 of 12

Proof According to Lemma 2.1 and $\left.t^{\alpha(1-\beta)} l(t)\right|_{t=0}=\phi_{p}\left(\int_{0}^{\tau} a(s) h(s) d s\right)$, problem (2.10) is equivalent to the following integral equation:

$$
\begin{aligned}
l(t)= & \phi_{p}\left[\int_{0}^{\tau} a(s) B l(s) d s\right] t^{\alpha(\beta-1)} \\
& +\frac{1}{\Gamma(\beta)} \int_{0}^{t}\left(\frac{t^{\alpha}-s^{\alpha}}{\alpha}\right)^{\beta-1} f\left(s, B l(s), \phi_{q}(l(s))\right) \frac{d s}{s^{1-\alpha}} \\
:= & A l(t) .
\end{aligned}
$$

For any $h, l \in C_{\alpha(1-\beta)}([0, d], \mathbb{R})$, we have

$$
\begin{aligned}
& \|A h-A l\|_{*} \\
& \leq \sup _{t \in[0, d]} t^{\alpha(\beta-1)}\left|\phi_{p}\left[\int_{0}^{\tau} a(s) B h(s) d s\right]-\phi_{p}\left[\int_{0}^{\tau} a(s) B l(s) d s\right]\right| \\
& +\sup _{t \in[0, d]} \frac{1}{\Gamma(\beta)} \int_{0}^{t}\left(\frac{t^{\alpha}-s^{\alpha}}{\alpha}\right)^{\beta-1}\left|f\left(s, B h(s), \phi_{q}(h(s))\right)-f\left(s, B l(s), \phi_{q}(l(s))\right)\right| \frac{d s}{s^{1-\alpha}} \\
& \leq \sup _{t \in[0, d]} t^{\alpha(\beta-1)} \mid \phi_{p}\left[\int_{0}^{\tau} a(s)\left(r s^{\alpha(\gamma-1)}+\frac{1}{\Gamma(\gamma)} \int_{0}^{s}\left(\frac{s^{\alpha}-u^{\alpha}}{\alpha}\right)^{\gamma-1} \phi_{q}(h(u)) \frac{d u}{u^{1-\alpha}}\right) d s\right] \\
& -\phi_{p}\left[\int_{0}^{\tau} a(s)\left(r s^{\alpha(\gamma-1)}+\frac{1}{\Gamma(\gamma)} \int_{0}^{s}\left(\frac{s^{\alpha}-u^{\alpha}}{\alpha}\right)^{\gamma-1} \phi_{q}(l(u)) \frac{d u}{u^{1-\alpha}}\right) d s\right] \mid \\
& +\sup _{t \in[0, d]} \frac{1}{\Gamma(\beta)} \int_{0}^{t}\left(\frac{t^{\alpha}-s^{\alpha}}{\alpha}\right)^{\beta-1} M(s)|h(s)-l(s)| \frac{d s}{s^{1-\alpha}} \\
& \leq \sup _{t \in[0, d]} t^{\alpha(\beta-1)} \mid\left[\int_{0}^{\tau} a(s)\left(r s^{\alpha(\gamma-1)}\right) d s\right. \\
& \left.\left.+\int_{0}^{\tau} a(s) \frac{1}{\Gamma(\gamma)} \int_{0}^{s}\left(\frac{s^{\alpha}-u^{\alpha}}{\alpha}\right)^{\gamma-1}\left|\phi_{q}(h(u))\right| \frac{d u}{u^{1-\alpha}}\right) d s\right]^{p-1} \\
& \left.-\left[\int_{0}^{\tau} a(s)\left(r s^{\alpha(\gamma-1)}\right) d s+\int_{0}^{\tau} a(s) \frac{1}{\Gamma(\gamma)} \int_{0}^{s}\left(\frac{s^{\alpha}-u^{\alpha}}{\alpha}\right)^{\gamma-1}\left|\phi_{q}(l(u))\right| \frac{d u}{u^{1-\alpha}}\right) d s\right]^{p-1} \mid \\
& +\sup _{t \in[0, d]} \frac{1}{\Gamma(\beta)} \int_{0}^{t}\left(\frac{t^{\alpha}-s^{\alpha}}{\alpha}\right)^{\beta-1} M(s) \frac{d s}{s^{1-\alpha}}\|h-l\|_{*} \\
& \leq \sup _{t \in[0, d]} t^{\alpha(\beta-1)} \mid(p-1) \xi^{p-2}\left[\int_{0}^{\tau} a(s)\left(r s^{\alpha(\gamma-1)}\right) d s+\int_{0}^{\tau} a(s) \frac{1}{\Gamma(\gamma)} \int_{0}^{s}\left(\frac{s^{\alpha}-u^{\alpha}}{\alpha}\right)^{\gamma-1}\right. \\
& \times \phi_{q}(h(u)) \frac{d u}{u^{1-\alpha}} d s-\int_{0}^{\tau} a(s)\left(r s^{\alpha(\gamma-1)}\right) d s \\
& \left.-\int_{0}^{\tau} a(s) \frac{1}{\Gamma(\gamma)} \int_{0}^{s}\left(\frac{s^{\alpha}-u^{\alpha}}{\alpha}\right)^{\gamma-1} \phi_{q}(l(u)) \frac{d u}{u^{1-\alpha}} d s\right] \mid \\
& +\sup _{t \in[0, d]} \frac{M}{\Gamma(\beta+1)}\left(\frac{t^{\alpha}}{\alpha}\right)^{\beta}\|h-l\|_{*} \\
& \leq d^{\alpha(\beta-1)}(p-1) \xi^{p-2}\left|\int_{0}^{\tau} a(s) \frac{1}{\Gamma(\gamma)} \int_{0}^{s}\left(\frac{s^{\alpha}-u^{\alpha}}{\alpha}\right)^{\gamma-1}\left(\phi_{q}(h(u))-\phi_{q}(l(u))\right) \frac{d u}{u^{1-\alpha}} d s\right|
\end{aligned}
$$




$$
\begin{aligned}
& +\frac{M d^{\alpha \beta}}{\Gamma(\beta+1) \alpha^{\beta}}\|h-l\|_{*} \\
\leq & d^{\alpha(\beta-1)}(p-1) \xi^{p-2}(q-1) \eta^{q-2} \int_{0}^{\tau} a(s) \frac{1}{\Gamma(\gamma)} \int_{0}^{s}\left(\frac{s^{\alpha}-u^{\alpha}}{\alpha}\right)^{\gamma-1} \frac{d u}{u^{1-\alpha}} d s\|h-l\|_{*} \\
& +\frac{M d^{\alpha \beta}}{\Gamma(\beta+1) \alpha^{\beta}}\|h-l\|_{*} \\
= & \left(\frac{d^{\alpha(\beta-1)} \xi^{p-2} \eta^{q-2}}{\Gamma(\gamma+1)} \int_{0}^{\tau} a(s)\left(\frac{s^{\alpha}}{\alpha}\right)^{\gamma-1} d s+\frac{M d^{\alpha \beta}}{\Gamma(\beta+1) \alpha^{\beta}}\right)\|h-l\|_{*},
\end{aligned}
$$

which, in view of (2.11), implies that the operator $A$ has a unique fixed point by the Banach fixed point theorem. In consequence, problem (2.10) has a unique solution.

Lemma 2.3 If $0<\alpha, \gamma, \beta<1, \psi \in C_{\alpha(1-\beta)}([0, d], \mathbb{R})$, and $M$ is a nonnegative bounded integrable function on $[0, d]$, then the following problem

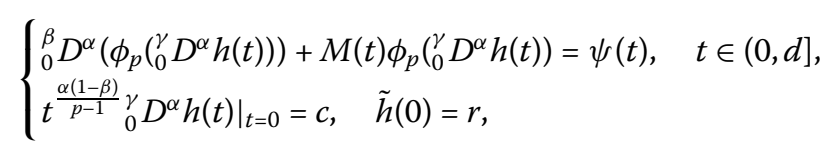

has a unique solution $h \in Y$, provided that $M d^{\alpha \beta}<\Gamma(\beta+1) \alpha^{\beta}$.

Proof Letting $l(t)=\phi_{p}\left({ }_{0}^{\gamma} D^{\alpha} h(t)\right)$, we have

$$
\left\{\begin{array}{l}
{ }_{0}^{\gamma} D^{\alpha} h(t)=\phi_{q}(l(t)), \quad t \in(0, d] \\
\tilde{h}(0)=r
\end{array}\right.
$$

and

$$
\left\{\begin{array}{l}
{ }_{0}^{\beta} D^{\alpha} l(t)+M(t) l(t)=\psi(t), \quad t \in(0, d] \\
\left.t^{\alpha(1-\beta)} l(t)\right|_{t=0}=\phi_{p}(c)
\end{array}\right.
$$

Let $f\left(t, B l(t), \phi_{q}(l(t))\right)=\psi(t)-M(t) l(t)$. For $l_{1}, l_{2} \in C_{\alpha(1-\beta)}([0, d], \mathbb{R})$, we have

$$
\left|f\left(t, B l_{1}, \phi_{q}\left(l_{1}\right)\right)-f\left(t, B l_{2}, \phi_{q}\left(l_{2}\right)\right)\right|=|M(t)|\left|l_{2}-l_{1}\right| \leq M\left|l_{2}-l_{1}\right| .
$$

Thus, problem (2.15) has a unique solution $l \in C_{\alpha(1-\beta)}([0, d], \mathbb{R})$ by Lemma 2.2, and ${ }_{0}^{\gamma} D^{\alpha} h \in C_{\frac{\alpha(1-\beta)}{p-1}}([0, d], \mathbb{R})$. Moreover, problem $(2.14)$ has a solution $h \in C_{\alpha(1-\gamma)}([0, d], \mathbb{R})$ by Lemma 2.1. By inserting $l$ in $h$, we get a unique solution $h \in Y$ of problem (2.13).

Definition 2.3 If $h \in Y$ is a lower solution of (1.1), then

$$
\left\{\begin{array}{l}
{ }_{0}^{\beta} D^{\alpha}\left(\phi_{p}\left({ }_{0}^{\gamma} D^{\alpha} h(t)\right)\right) \leq f\left(t, h(t),{ }_{0}^{\gamma} D^{\alpha} h(t)\right), \quad t \in(0, d], d>0, \\
\left.t^{\frac{\alpha(1-\beta)}{p-1}}{ }_{0}^{\gamma} D^{\alpha} h(t)\right|_{t=0} \leq \int_{0}^{\tau} a(s) h(s) d s, \quad g(\tilde{h}(0), \tilde{h}(d)) \leq 0, \tau \in(0, d) .
\end{array}\right.
$$

If $l \in Y$ is an upper solution of (1.1), then

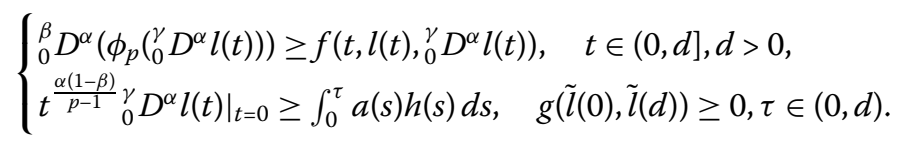




\section{Lemma 2.4 (Comparison theorem)}

$\left(C_{1}\right)$ Let $M$ be a nonnegative bounded integrable function on $[0, d]$. If $m \in C_{\alpha(1-\beta)}([0, d], \mathbb{R})$ satisfies

$$
\left\{\begin{array}{l}
{ }_{0}^{\beta} D^{\alpha} m(t)+M(t) m(t) \geq 0, \quad t \in(0, d] \\
\left.t^{\alpha(1-\beta)} m(t)\right|_{t=0} \geq 0
\end{array}\right.
$$

then $m(t) \geq 0, t \in(0, d]$.

$\left(C_{2}\right)$ Assume that $n \in C_{\alpha(1-\gamma)}([0, d], \mathbb{R})$ satisfies

$$
\left\{\begin{array}{l}
{ }_{0}^{\gamma} D^{\alpha} n(t) \geq 0, \quad t \in(0, d], \\
\left.t^{\alpha(1-\gamma)} n(t)\right|_{t=0} \geq 0
\end{array}\right.
$$

Then $n(t) \geq 0, t \in(0, d]$.

Proof Assume that $m(t) \geq 0$ is not true. Then there exist $t_{1}, t_{2} \in(0, d]$ such that $m\left(t_{2}\right)<0$, $m\left(t_{1}\right)=0$ and $m(t) \geq 0$ for $t \in\left(0, t_{1}\right)$ and $m(t)<0$ for $t \in\left(t_{1}, t_{2}\right)$. Since $M(t) \geq 0, \forall t \in[0, d]$, we have ${ }_{0}^{\beta} D^{\alpha} m(t) \geq 0, \forall t \in\left(t_{1}, t_{2}\right)$.

According to

$$
{ }_{0}^{\beta} D^{\alpha} m(t)=t^{1-\alpha} \frac{d}{d t}{ }^{1-\beta} I^{\alpha} m(t)
$$

we obtain that ${ }_{0}^{1-\beta} I^{\alpha} m(t)$ is nondecreasing on $\left(t_{1}, t_{2}\right)$. Hence ${ }_{0}^{1-\beta} I^{\alpha} m(t)-{ }_{0}^{1-\beta} I^{\alpha} m\left(t_{1}\right) \geq 0$, $t \in\left(t_{1}, t_{2}\right)$. On the other hand, we have

$$
\begin{aligned}
{ }_{0}^{1-\beta} I^{\alpha} m(t)-{ }_{0}^{1-\beta} I^{\alpha} m\left(t_{1}\right) \\
=\frac{1}{\Gamma(1-\beta)} \int_{0}^{t}\left(\frac{t^{\alpha}-s^{\alpha}}{\alpha}\right)^{-\beta} m(s) \frac{d s}{s^{1-\alpha}}-\frac{1}{\Gamma(1-\beta)} \int_{0}^{t_{1}}\left(\frac{t_{1}^{\alpha}-s^{\alpha}}{\alpha}\right)^{-\beta} m(s) \frac{d s}{s^{1-\alpha}} \\
=\frac{1}{\Gamma(1-\beta)} \int_{0}^{t_{1}}\left[\left(\frac{t^{\alpha}-s^{\alpha}}{\alpha}\right)^{-\beta}-\left(\frac{t_{1}^{\alpha}-s^{\alpha}}{\alpha}\right)^{-\beta}\right] m(s) \frac{d s}{s^{1-\alpha}} \\
\quad+\frac{1}{\Gamma(1-\beta)} \int_{t_{1}}^{t}\left(\frac{t^{\alpha}-s^{\alpha}}{\alpha}\right)^{-\beta} m(s) \frac{d s}{s^{1-\alpha}} \\
<0, \quad \forall t \in\left(t_{1}, t_{2}\right),
\end{aligned}
$$

which is a contradiction. Therefore, $m(t) \geq 0, \forall t \in(0, d]$.

Obviously, the conclusion of $\left(C_{2}\right)$ holds. It follows from $(2.8)$ that $n(t) \geq 0, \forall t \in(0, d]$.

\section{Main results}

\section{Theorem 3.1 Assume that}

$\left(L_{1}\right) h_{0}, l_{0} \in Y$ are lower and upper solutions of (1.1), respectively with $h_{0}(t) \leq l_{0}(t), t \in$ $(0, d]$

$\left(L_{2}\right)$ there exists a function $M \in C([0, d], \mathbb{R}), t \in[0, d]$ such that

$$
f\left(t, l(t),{ }_{0}^{\gamma} D^{\alpha} l(t)\right)-f\left(t, h(t),{ }_{0}^{\gamma} D^{\alpha} h(t)\right) \geq-M(t)\left[\phi_{p}\left({ }_{0}^{\gamma} D^{\alpha} l(t)\right)-\phi_{p}\left({ }_{0}^{\gamma} D^{\alpha} h(t)\right)\right]
$$

$$
\text { for } h_{0}(t) \leq h(t) \leq l(t) \leq l_{0}(t), t \in(0, d] \text {; }
$$


$\left(L_{3}\right)$ the function $g$ satisfies

$$
\begin{gathered}
\quad g\left(m_{2}, n_{2}\right)-g\left(m_{1}, n_{1}\right) \geq m_{2}-m_{1} \\
\text { for } \tilde{h}_{0}(0) \leq m_{2} \leq m_{1} \leq \tilde{l}_{0}(0), \tilde{h}_{0}(d) \leq n_{2} \leq n_{1} \leq \tilde{l}_{0}(d), \text { if } M(t) d^{\alpha \beta}<\Gamma(\beta+1) \alpha^{\beta} .
\end{gathered}
$$

Then there exist sequences $\left\{h_{n}\right\},\left\{l_{n}\right\} \in Y$ such that (1.1) has extremal solutions $m(t), n(t)$ in $\left[h_{0}, l_{0}\right]=\left\{h \in Y: h_{0}(t) \leq h(t) \leq l_{0}(t), t \in(0, d]\right\}$ satisfying

$$
\left\{\begin{array}{c}
h_{0}(t) \leq h_{1}(t) \leq \cdots \leq h_{n}(t) \leq \cdots \leq m(t) \leq n(t) \leq \cdots \leq l_{n}(t) \leq \cdots \leq l_{1}(t) \leq l_{0}(t), \\
{ }_{0}^{\gamma} D^{\alpha} h_{0} \leq{ }_{0}^{\gamma} D^{\alpha} h_{1} \leq \cdots \leq{ }_{0}^{\gamma} D^{\alpha} h_{n} \leq \cdots \leq{ }_{0}^{\gamma} D^{\alpha} m \leq{ }_{0}^{\gamma} D^{\alpha} n \leq \cdots \\
\quad \leq{ }_{0}^{\gamma} D^{\alpha} l_{n} \leq \cdots \leq{ }_{0}^{\gamma} D^{\alpha} l_{1} \leq{ }_{0}^{\gamma} D^{\alpha} l_{0}, \\
\phi_{p}\left({ }_{0}^{\gamma} D^{\alpha} h_{0}\right) \leq \phi_{p}\left({ }_{0}^{\gamma} D^{\alpha} h_{1}\right) \leq \cdots \leq \phi_{p}\left({ }_{0}^{\gamma} D^{\alpha} h_{n}\right) \leq \cdots \leq \phi_{p}\left({ }_{0}^{\gamma} D^{\alpha} m\right) \leq \phi_{p}\left({ }_{0}^{\gamma} D^{\alpha} n\right) \leq \cdots \\
\leq \phi_{p}\left({ }_{0}^{\gamma} D^{\alpha} l_{n}\right) \leq \cdots \leq \phi_{p}\left({ }_{0}^{\gamma} D^{\alpha} l_{1}\right) \leq \phi_{p}\left({ }_{0}^{\gamma} D^{\alpha} l_{0}\right),
\end{array}\right.
$$

for $t \in(0, d], n=1,2,3, \ldots$

Proof Let $F(h(t))=f\left(t, h(t),{ }_{0}^{\gamma} D^{\alpha} h(t)\right)$. For $n=1,2, \ldots$, we define

$$
\left\{\begin{array}{l}
{ }_{0}^{\beta} D^{\alpha}\left(\phi_{p}\left({ }_{0}^{\gamma} D^{\alpha} h_{n}(t)\right)\right)+M(t) \phi_{p}\left({ }_{0}^{\gamma} D^{\alpha} h_{n}(t)\right) \\
\quad=F\left(h_{n-1}(t)\right)+M(t) \phi_{p}\left({ }_{0}^{\gamma} D^{\alpha} h_{n-1}(t)\right), \quad t \in(0, d], \\
\left.t^{\frac{\alpha(1-\beta)}{p-1}}{ }_{0} D^{\alpha} h_{n}(t)\right|_{t=0}=\int_{0}^{\tau} a(s) h_{n-1}(s) d s, \\
\tilde{h}_{n}(0)=\tilde{h}_{n-1}(0)-g\left(\tilde{h}_{n-1}(0), \tilde{h}_{n-1}(d)\right), \quad \tau \in(0, d),
\end{array}\right.
$$

and

$$
\left\{\begin{array}{l}
{ }_{0}^{\beta} D^{\alpha}\left(\phi_{p}\left({ }_{0}^{\gamma} D^{\alpha} l_{n}(t)\right)\right)+M(t) \phi_{p}\left({ }_{0}^{\gamma} D^{\alpha} l_{n}(t)\right) \\
\quad=F\left(l_{n-1}(t)\right)+M(t) \phi_{p}\left({ }_{0}^{\gamma} D^{\alpha} l_{n-1}(t)\right), \quad t \in(0, d], \\
\left.t^{\frac{\alpha(1-\beta)}{p-1}}{ }_{0}^{\gamma} D^{\alpha} l_{n}(t)\right|_{t=0}=\int_{0}^{\tau} a(s) l_{n-1}(s) d s, \\
\tilde{l}_{n}(0)=\tilde{l}_{n-1}(0)-g\left(\tilde{l}_{n-1}(0), \tilde{l}_{n-1}(d)\right), \quad \tau \in(0, d) .
\end{array}\right.
$$

Notice that the functions $h_{1}, l_{1}$ are well defined in $Y$ by Lemma 2.3.

Now, we prove that $h_{0}(t) \leq h_{1}(t) \leq l_{1}(t) \leq l_{0}(t),{ }_{0}^{\gamma} D^{\alpha} h_{0}(t) \leq{ }_{0}^{\gamma} D^{\alpha} h_{1}(t) \leq{ }_{0}^{\gamma} D^{\alpha} l_{1}(t) \leq$ ${ }_{0}^{\gamma} D^{\alpha} l_{0}(t), t \in(0, d]$, and $\tilde{h}_{0}(0) \leq \tilde{h}_{1}(0) \leq \tilde{l}_{1}(0) \leq \tilde{l}_{0}(0)$. Let $\lambda(t)=\phi_{p}\left({ }_{0}^{\gamma} D^{\alpha} h_{1}(t)\right)-\phi_{p}\left({ }_{0}^{\gamma} D^{\alpha} h_{0}(t)\right)$. From (2.9), (3.1), and $\left(L_{1}\right)$, we have

$$
\left\{\begin{array}{c}
{ }_{0}^{\beta} D^{\alpha} \lambda(t)+M(t) \lambda(t)=F\left(h_{0}(t)\right)-{ }_{0}^{\beta} D^{\alpha}\left(\phi_{p}\left({ }_{0}^{\gamma} D^{\alpha} h_{0}(t)\right)\right) \geq 0, \\
\left.t^{\alpha(1-\beta)} \lambda(t)\right|_{t=0}=\left.\phi_{p}\left(t^{\frac{\alpha(1-\beta)}{p-1}}{ }_{0} D^{\alpha} h_{1}(t)\right)\right|_{t=0}-\left.\phi_{p}\left(t^{\frac{\alpha(1-1)}{p-1}}{ }_{0} D^{\alpha} h_{0}(t)\right)\right|_{t=0} \\
\geq \int_{0}^{\tau} a(s) h_{0}(s) d s-\int_{0}^{\tau} a(s) h_{0}(s) d s=0 .
\end{array}\right.
$$

By $\left(C_{1}\right)$ of Lemma 2.4, we obtain $\lambda(t) \geq 0, t \in(0, d]$, which means $\phi_{p}\left({ }_{0}^{\gamma} D^{\alpha} h_{1}(t)\right) \geq$ $\phi_{p}\left({ }_{0}^{\gamma} D^{\alpha} h_{0}(t)\right)$. The monotone increasing property of $\phi_{p}(t)$ ensures that ${ }_{0}^{\gamma} D^{\alpha} h_{1}(t) \geq$ ${ }_{0}^{\gamma} D^{\alpha} h_{0}(t)$. Thus, ${ }_{0}^{\gamma} D^{\alpha}\left(h_{1}(t)-h_{0}(t)\right) \geq 0$. According to $\tilde{h}_{1}(0)-\tilde{h}_{0}(0)=-g\left(\tilde{h}_{0}(0), \tilde{h}_{0}(d)\right) \geq 0$, we have $h_{1}(t) \geq h_{0}(t), t \in(0, d]$ by $\left(C_{2}\right)$ of Lemma 2.4. In a similar manner, we can obtain that $l_{1}(t) \leq l_{0}(t),{ }_{0}^{\gamma} D^{\alpha} h_{1}(t) \leq{ }_{0}^{\gamma} D^{\alpha} h_{0}(t), t \in(0, d]$, and $\tilde{l}_{1}(0) \leq \tilde{l}_{0}(0)$. 
Setting $\eta(t)=\phi_{p}\left({ }_{0}^{\gamma} D^{\alpha} l_{1}(t)\right)-\phi_{p}\left({ }_{0}^{\gamma} D^{\alpha} h_{1}(t)\right)$ and using $\left(L_{2}\right)$, we have

$$
\left\{\begin{array}{l}
{ }_{0}^{\beta} D^{\alpha} \eta(t)+M(t) \eta(t)=F\left(l_{0}(t)\right)-F\left(h_{0}(t)\right)+M(t)\left[\phi_{p}\left({ }_{0}^{\gamma} D^{\alpha} l_{0}(t)\right)-\phi_{p}\left({ }_{0}^{\gamma} D^{\alpha} h_{0}(t)\right)\right] \geq 0, \\
\left.t^{\alpha(1-\beta)} \eta(t)\right|_{t=0}=\left.\phi_{p}\left(t^{\frac{\alpha(1-\beta)}{p-1}}{ }_{0} D^{\alpha} l_{1}(t)\right)\right|_{t=0}-\left.\phi_{p}\left(t^{\frac{\alpha(1-\beta)}{p-1}}{ }_{0} D^{\alpha} h_{1}(t)\right)\right|_{t=0} \geq 0 .
\end{array}\right.
$$

By $\left(C_{1}\right)$ of Lemma 2.4, we obtain $\eta(t) \geq 0, t \in(0, d]$. Then $\phi_{p}\left({ }_{0}^{\gamma} D^{\alpha} l_{1}(t)\right) \geq \phi_{p}\left({ }_{0}^{\gamma} D^{\alpha} h_{1}(t)\right)$, and ${ }_{0}^{\gamma} D^{\alpha} l_{1}(t) \geq{ }_{0}^{\gamma} D^{\alpha} h_{1}(t)$. By $\left(L_{3}\right)$, we have

$$
\begin{aligned}
\tilde{l}_{1}(0)-\tilde{h}_{1}(0) & =\tilde{l}_{0}(0)-g\left(\tilde{l}_{0}(0), \tilde{l}_{0}(d)\right)-\tilde{h}_{0}(0)+g\left(\tilde{h}_{0}(0), \tilde{h}_{0}(d)\right) \\
& =\tilde{l}_{0}(0)-\tilde{h}_{0}(0)+g\left(\tilde{h}_{0}(0), \tilde{h}_{0}(d)\right)-g\left(\tilde{l}_{0}(0), \tilde{l}_{0}(d)\right) \\
& \geq \tilde{l}_{0}(0)-\tilde{h}_{0}(0)+\tilde{h}_{0}(0)-\tilde{l}_{0}(0)=0 .
\end{aligned}
$$

Thus, $l_{1}(t) \geq h_{1}(t), t \in(0, d]$ by $\left(C_{2}\right)$ of Lemma 2.4 .

Next, we show that $h_{1}, l_{1}$ are lower and upper solutions of (1.1), respectively. By (3.1) and $\left(L_{2}\right)$, we obtain

$$
\begin{aligned}
{ }_{0}^{\beta} D^{\alpha} & \left(\phi_{p}\left({ }_{0}^{\gamma} D^{\alpha} h_{1}(t)\right)\right) \\
= & F\left(h_{0}(t)\right)-M(t)\left[\phi_{p}\left({ }_{0}^{\gamma} D^{\alpha} h_{1}(t)\right)-\phi_{p}\left(\begin{array}{l}
\gamma \\
0
\end{array} D^{\alpha} h_{0}(t)\right)\right]-F\left(h_{1}(t)\right)+F\left(h_{1}(t)\right) \\
\leq & M(t)\left[\phi_{p}\left(\begin{array}{l}
\gamma \\
0
\end{array} D^{\alpha} h_{1}(t)\right)-\phi_{p}\left(\begin{array}{l}
\gamma \\
0
\end{array} D^{\alpha} h_{0}(t)\right)\right]-M(t)\left[\phi_{p}\left({ }_{0}^{\gamma} D^{\alpha} h_{1}(t)\right)\right. \\
& \left.-\phi_{p}\left({ }_{0}^{\gamma} D^{\alpha} h_{0}(t)\right)\right]+F\left(h_{1}(t)\right) \\
= & F\left(h_{1}(t)\right) .
\end{aligned}
$$

By $\left(L_{3}\right)$, we have

$$
\begin{aligned}
0 & =g\left(\tilde{h}_{0}(0), \tilde{h}_{0}(d)\right)-g\left(\tilde{h}_{1}(0), \tilde{h}_{1}(d)\right)+g\left(\tilde{h}_{1}(0), \tilde{h}_{1}(d)\right)+\tilde{h}_{1}(0)-\tilde{h}_{0}(0) \\
& \geq \tilde{h}_{0}(0)-\tilde{h}_{1}(0)+g\left(\tilde{h}_{1}(0), \tilde{h}_{1}(d)\right)+\tilde{h}_{1}(0)-\tilde{h}_{0}(0) \\
& =g\left(\tilde{h}_{1}(0), \tilde{h}_{1}(d)\right)
\end{aligned}
$$

and

$$
\left.t^{\frac{\alpha(1-\beta)}{p-1} \gamma} D^{\alpha} h_{1}(t)\right|_{t=0}=\int_{0}^{\tau} a(s) h_{0}(s) d s \leq \int_{0}^{\tau} a(s) h_{1}(s) d s,
$$

which imply that $h_{1}$ is a lower solution of (1.1). Analogously, we can verify that $l_{1}$ is an upper solution of (1.1).

Using the mathematical induction, we have

$$
\begin{aligned}
& h_{0}(t) \leq h_{1}(t) \leq \cdots \leq h_{n}(t) \leq h_{n+1}(t) \leq l_{n+1}(t) \leq l_{n}(t) \leq \cdots \leq l_{1}(t) \leq l_{0}(t) \text {, } \\
& { }_{0}^{\gamma} D^{\alpha} h_{0} \leq{ }_{0}^{\gamma} D^{\alpha} h_{1} \leq \cdots \leq{ }_{0}^{\gamma} D^{\alpha} h_{n} \leq{ }_{0}^{\gamma} D^{\alpha} h_{n+1} \leq{ }_{0}^{\gamma} D^{\alpha} l_{n+1} \leq{ }_{0}^{\gamma} D^{\alpha} l_{n} \leq \cdots \\
& \leq{ }_{0}^{\gamma} D^{\alpha} l_{1} \leq{ }_{0}^{\gamma} D^{\alpha} l_{0} \\
& \tilde{h}_{0}(0) \leq \tilde{h}_{1}(0) \leq \cdots \leq \tilde{h}_{n}(0) \leq \tilde{h}_{n+1}(0) \leq \tilde{l}_{n+1}(0) \leq \tilde{l}_{n}(0) \leq \cdots \leq \tilde{l}_{1}(0) \leq \tilde{l}_{0}(0)
\end{aligned}
$$


By the standard analysis, we can get that the sequences $\left\{t^{\alpha(1-\gamma)} h_{n}\right\}$ and $\left\{t^{\alpha(1-\gamma)} h_{n}\right\}$ are uniformly bounded and equicontinuous. Thus, in view of Arzela-Ascoli theorem, we obtain

$$
\begin{aligned}
& \lim _{n \rightarrow \infty} h_{n}(t)=m(t), \quad \lim _{n \rightarrow \infty} l_{n}(t)=n(t), \quad t \in(0, d], \\
& \lim _{n \rightarrow \infty}{ }_{0}^{\gamma} D^{\alpha} h_{n}(t)={ }_{0}^{\gamma} D^{\alpha} m(t), \quad \lim _{n \rightarrow \infty}{ }_{0}^{\gamma} D^{\alpha} l_{n}(t)={ }_{0}^{\gamma} D^{\alpha} n(t), \quad t \in(0, d] .
\end{aligned}
$$

Hence, $h_{0}(t) \leq m(t) \leq n(t) \leq l_{0}(t)$ on $(0, d]$ and $m(t), n(t)$ are solutions of (1.1).

Moreover, we show that $m(t), n(t)$ are extremal solutions of (1.1). Let $h \in\left[h_{0}, l_{0}\right]$ be any solution of (1.1). Let $h_{n}(t) \leq h(t) \leq l_{n}(t), t \in(0, d]$ and that

$$
j(t)=\phi_{p}\left({ }_{0}^{\gamma} D^{\alpha} h(t)\right)-\phi_{p}\left({ }_{0}^{\gamma} D^{\alpha} h_{n+1}(t)\right), \quad k(t)=\phi_{p}\left({ }_{0}^{\gamma} D^{\alpha} l_{n+1}(t)\right)-\phi_{p}\left({ }_{0}^{\gamma} D^{\alpha} h(t)\right) .
$$

By $\left(L_{2}\right)$, we obtain

$$
\left\{\begin{array}{l}
{ }_{0}^{\beta} D^{\alpha} j(t)+M(t) j(t)=F(h(t))-F\left(h_{n}(t)\right)+M(t)\left[\phi_{p}\left({ }_{0}^{\gamma} D^{\alpha} h(t)\right)-\phi_{p}\left({ }_{0}^{\gamma} D^{\alpha} h_{n}(t)\right)\right] \geq 0, \\
\left.t^{\alpha(1-\beta)} j(t)\right|_{t=0}=\left.\phi_{p}\left(t^{\frac{\alpha(1-\beta)}{p-1}}{ }_{0} D^{\alpha} h(t)\right)\right|_{t=0}-\left.\phi_{p}\left(t^{\frac{\alpha(1-\beta)}{p-1}}{ }_{0} D^{\alpha} h_{n+1}(t)\right)\right|_{t=0} \geq 0,
\end{array}\right.
$$

and

$$
\left\{\begin{array}{l}
{ }_{0}^{\beta} D^{\alpha} k(t)+M(t) k(t)=F\left(l_{n}(t)\right)-F(h(t))+M(t)\left[\phi_{p}\left({ }_{0}^{\gamma} D^{\alpha} l_{n}(t)\right)-\phi_{p}\left({ }_{0}^{\gamma} D^{\alpha} h(t)\right)\right] \geq 0, \\
\left.t^{\alpha(1-\beta)} k(t)\right|_{t=0}=\left.\phi_{p}\left(t^{\frac{\alpha(1-\beta)}{p-1}} \gamma_{0}^{\gamma} D^{\alpha} l_{n+1}(t)\right)\right|_{t=0}-\left.\phi_{p}\left(t^{\frac{\alpha(1-\beta)}{p-1}}{ }_{0}^{\gamma} D^{\alpha} h(t)\right)\right|_{t=0} \geq 0 .
\end{array}\right.
$$

Thus, by $\left(C_{1}\right)$ of Lemma 2.4, we have $j(t) \geq 0, k(t) \geq 0$. Then $\phi_{p}\left({ }_{0}^{\gamma} D^{\alpha} h(t)\right) \geq \phi_{p}\left({ }_{0}^{\gamma} D^{\alpha} h_{n+1}(t)\right)$, $\phi_{p}\left({ }_{0}^{\gamma} D^{\alpha} l_{n+1}(t)\right) \geq \phi_{p}\left({ }_{0}^{\gamma} D^{\alpha} h(t)\right)$. Hence, ${ }_{0}^{\gamma} D^{\alpha}\left(h(t)-h_{n+1}(t)\right) \geq 0,{ }_{0}^{\gamma} D^{\alpha}\left(l_{n+1}(t)-h(t)\right) \geq 0$.

By $\left(L_{3}\right)$, we have

$$
\begin{aligned}
\tilde{h}(0)-\tilde{h}_{n+1}(0) & =\tilde{h}(0)-\tilde{h}_{n}(0)+g\left(\tilde{h}_{n}(0), \tilde{h}_{n}(d)\right)-g(\tilde{h}(0), \tilde{h}(d)) \\
& \geq \tilde{h}(0)-\tilde{h}_{n}(0)+\tilde{h}_{n}(0)-\tilde{h}(0) \\
& =0
\end{aligned}
$$

and

$$
\begin{aligned}
\tilde{l}_{n+1}(0)-\tilde{h}(0) & =\tilde{l}_{n}(0)-\tilde{h}(0)-g\left(\tilde{l}_{n}(0), \tilde{l}_{n}(d)\right)+g(\tilde{h}(0), \tilde{h}(d)) \\
& \geq \tilde{l}_{n}(0)-\tilde{h}(0)+\tilde{h}(0)-\tilde{l}_{n}(0) \\
& =0 .
\end{aligned}
$$

Hence, $h_{n+1}(t) \leq h(t) \leq l_{n+1}(t), t \in(0, d]$ by $\left(C_{2}\right)$ of Lemma 2.4, which, on taking the limit $n \rightarrow \infty$, yields $m(t) \leq h(t) \leq n(t)$. Therefore, $m(t), n(t)$ are extremal solutions of (1.1).

Theorem 3.2 If the hypotheses of Theorem 3.1 hold, $a(t)=0$, and there exists a function $L(t) \geq 0$ such that

$$
L(t)\left[\phi_{p}\left({ }_{0}^{\gamma} D^{\alpha} l(t)\right)-\phi_{p}\left({ }_{0}^{\gamma} D^{\alpha} h(t)\right)\right] \leq f\left(t, h(t),{ }_{0}^{\gamma} D^{\alpha} h(t)\right)-f\left(t, l(t),{ }_{0}^{\gamma} D^{\alpha} l(t)\right)
$$


for $h_{0}(t) \leq h(t) \leq l(t) \leq l_{0}(t), t \in(0, d]$ and $\tilde{h}_{0}(0)=\tilde{l}_{0}(0)$, then (1.1) has a unique solution in $\left[h_{0}, l_{0}\right]$.

Proof It follows by Theorem 3.1 that $m(t)$ and $n(t)$ are extremal solutions such that $m(t) \leq n(t), t \in(0, d]$. Then we just need to prove $m(t) \geq n(t), t \in(0, d]$. Letting $\lambda(t)=$ $\phi_{p}\left({ }_{0}^{\gamma} D^{\alpha} m(t)\right)-\phi_{p}\left({ }_{0}^{\gamma} D^{\alpha} n(t)\right), t \in(0, d]$ and using (3.5), we obtain

$$
\left\{\begin{array}{l}
{ }_{0}^{\beta} D^{\alpha} \lambda(t)=F(m(t))-F(n(t)) \geq L(t)\left[\phi_{p}\left({ }_{0}^{\gamma} D^{\alpha} n(t)\right)-\phi_{p}\left({ }_{0}^{\gamma} D^{\alpha} m(t)\right)\right]=-L(t) \lambda(t), \\
\left.t^{\alpha(1-\beta)} \lambda(t)\right|_{t=0}=\left.\phi_{p}\left(t^{\frac{\alpha(1-\beta)}{p-1}}{ }_{0} D^{\alpha} m(t)\right)\right|_{t=0}-\left.\phi_{p}\left(t^{\frac{\alpha(1-\beta)}{p-1}}{ }_{0}^{\gamma} D^{\alpha} n(t)\right)\right|_{t=0}=0 .
\end{array}\right.
$$

Then, by $\left(C_{1}\right)$ of Lemma 2.4, we have $\lambda(t) \geq 0$. Thus, $\phi_{p}\left({ }_{0}^{\gamma} D^{\alpha} m(t)\right) \geq \phi_{p}\left({ }_{0}^{\gamma} D^{\alpha} n(t)\right)$. Since $\phi_{p}(t)$ is nondecreasing, we have ${ }_{0}^{\gamma} D^{\alpha} m(t) \geq{ }_{0}^{\gamma} D^{\alpha} n(t), t \in(0, d]$. Then, by $\left(C_{2}\right)$ of Lemma 2.4, we obtain $m(t) \geq n(t)$. Furthermore, we have $\tilde{m}(0)=\tilde{n}(0)$ by $\tilde{h}_{0}(0)=\tilde{l}_{0}(0)$ and (3.4). Therefore, we have $m=n$. The proof is completed.

\section{Example}

Consider the following problem:

$$
\left\{\begin{array}{l}
{ }_{0}^{\frac{2}{3}} D^{\frac{1}{2}}\left(\phi_{3}\left({ }_{0}^{\frac{1}{2}} D^{\frac{1}{2}} h(t)\right)\right)=f\left(t, h(t),{ }_{0}^{\frac{1}{2}} D^{\frac{1}{2}} h(t)\right), \quad t \in(0,1], \\
\left.t t^{\frac{1}{12}}{ }_{0}^{\frac{1}{2}} D^{\frac{1}{2}} h(t)\right|_{t=0}=\int_{0}^{\tau} a(s) h(s) d s, \quad \frac{1}{2} \tilde{h}(0)-3 \tilde{h}(0) \tilde{h}(1)=0,
\end{array}\right.
$$

where $\alpha=\frac{1}{2}, \gamma=\frac{1}{2}, \beta=\frac{2}{3}, d=1, p=3, a(t)=0, \tau=1$, and $f\left(t, h(t),{ }_{0}^{\frac{1}{2}} D^{\frac{1}{2}} h(t)\right)=\frac{1}{2} t+h(t)-$ $2_{0}^{\frac{1}{2}} D^{\frac{1}{2}} h(t), g(m, n)=\frac{1}{2} m-3 m n$. Let $h_{0}(t)=0, l_{0}(t)=\Gamma\left(\frac{1}{2}\right) t^{\frac{1}{2}}$. Then we have ${ }_{0}^{\frac{1}{2}} D^{\frac{1}{2}} h_{0}(t)=0$, ${ }_{0}^{\frac{1}{2}} D^{\frac{1}{2}} l_{0}(t)=2^{\frac{1}{2}} t^{\frac{1}{4}}$, and

$$
\begin{aligned}
& { }_{0}^{\frac{2}{3}} D^{\frac{1}{2}}\left(\phi_{3}\left({ }_{0}^{\frac{1}{2}} D^{\frac{1}{2}} h_{0}(t)\right)\right)=0 \leq \frac{1}{2} t=f\left(t, h_{0}(t),{ }_{0}^{\frac{1}{2}} D^{\frac{1}{2}} h_{0}(t)\right), \quad t \in(0,1], \\
& \left.t^{\frac{1}{12}}{ }_{0}^{\frac{1}{2}} D^{\frac{1}{2}} h_{0}(t)\right|_{t=0}=0, \quad g\left(\tilde{h}_{0}(0), \tilde{h}_{0}(1)\right)=0, \\
& { }_{0}^{\frac{2}{3}} D^{\frac{1}{2}}\left(\phi_{3}\left({ }_{0}^{\frac{1}{2}} D^{\frac{1}{2}} l_{0}(t)\right)\right)= \\
& =f\left(t, l_{0}^{\frac{2}{3}} D^{\frac{1}{2}}\left(2 t^{\frac{1}{2}}\right)=\frac{3 \cdot 2^{\frac{1}{3}}}{\Gamma\left(\frac{1}{3}\right)} t_{0}^{\frac{1}{6}} \geq \frac{1}{2} t+\Gamma\left(\frac{1}{2}\right) t^{\frac{1}{2}}-2^{\frac{1}{2}} t_{0}^{\frac{1}{4}}(t)\right), \\
& \left.t^{\frac{1}{12}} D^{\frac{1}{2}} D^{\frac{1}{2}} l_{0}(t)\right|_{t=0}=0, \quad g\left(\tilde{l}_{0}(0), \tilde{l}_{0}(1)\right)=0 .
\end{aligned}
$$

Thus, $h_{0}$ and $l_{0}$ are lower and upper solutions of (4.1), respectively, and $h_{0} \leq l_{0}$ on $[0,1]$. In addition, for $h_{0} \leq h \leq l \leq l_{0}$, we have

$$
\begin{aligned}
f(t, & \left.h(t),{ }_{0}^{\frac{1}{2}} D^{\frac{1}{2}} h(t)\right)-f\left(t, l(t),{ }_{0}^{\frac{1}{2}} D^{\frac{1}{2}} l(t)\right) \\
& =h(t)-l(t)-2_{0}^{\frac{1}{2}} D^{\frac{1}{2}} h(t)+2_{0}^{\frac{1}{2}} D^{\frac{1}{2}} l(t) \\
& \leq 2\left[{ }_{0}^{\frac{1}{2}} D^{\frac{1}{2}} l(t)-{ }_{0}^{\frac{1}{2}} D^{\frac{1}{2}} h(t)\right] \\
& \leq M(t)\left[\phi_{3}\left({ }_{0}^{\frac{1}{2}} D^{\frac{1}{2}} l(t)\right)-\phi_{3}\left({ }_{0}^{\frac{1}{2}} D^{\frac{1}{2}} h(t)\right)\right],
\end{aligned}
$$

where $M(t)=2$. 
For $\tilde{h}_{0}(0) \leq m_{2} \leq m_{1} \leq \tilde{l}_{0}(0), \tilde{h}_{0}(1) \leq n_{2} \leq n_{1} \leq \tilde{l}_{0}(1)$, we have

$$
\begin{aligned}
g\left(m_{1}, n_{1}\right)-g\left(m_{2}, n_{2}\right) & =\frac{1}{2} m_{1}-3 m_{1} n_{1}-\frac{1}{2} m_{2}+3 m_{2} n_{2} \\
& \leq \frac{1}{2}\left(m_{1}-m_{2}\right) \leq m_{1}-m_{2} .
\end{aligned}
$$

Hence, assumptions $\left(L_{1}\right),\left(L_{2}\right)$, and $\left(L_{3}\right)$ hold. According to Theorem 3.1, there exist monotone iterative sequences $\left\{h_{n}\right\},\left\{l_{n}\right\}$ such that $\lim _{n \rightarrow \infty} h_{n}=m, \lim _{n \rightarrow \infty} l_{n}=n$ on $(0,1]$ and $m$, $n$ are the extremal solutions on $\left[h_{0}, l_{0}\right]$ of $(4.1)$.

\section{Acknowledgements}

The authors would like to express all gratitude to the anonymous referees for their hard work, helpful comments, and suggestions.

\section{Funding}

The work is supported by NSFC (No. 11501342), NSF of Shanxi, China (No. 201701D221007), and STIP (Nos. 201802068 and 201802069)

Availability of data and materials

Data sharing not applicable to this article as no datasets were generated or analysed during the current study.

\section{Competing interests}

The authors declare that they have no competing interests.

\section{Authors' contributions}

All authors equally contributed this manuscript and approved the final version.

\section{Author details}

${ }^{1}$ School of Mathematics and Computer Science, Shanxi Normal University, Linfen, People's Republic of China. ${ }^{2}$ NAAM Research Group, Faculty of Science, King Abdulaziz University, Jeddah, Saudi Arabia.

\section{Publisher's Note}

Springer Nature remains neutral with regard to jurisdictional claims in published maps and institutional affiliations.

Received: 5 April 2019 Accepted: 13 August 2019 Published online: 02 September 2019

\section{References}

1. Miller, K.S., Ross, B.: An Introduction to the Fractional Calculus and Fractional Differential Equations. Wiley, New York (1993)

2. Podlubny, I.: Fractional Differential Equations. Academic Press, New York (1999)

3. Kilbas, A.A., Srivastava, H.M., Trujillo, J.J.: Theory and Applications of Fractional Differential Equations. Elsevier, Amsterdam (2006)

4. Ahmad, B., Alsaedi, A., Ntouyas, S.K., Tariboon, J.: Hadamard-Type Fractional Differential Equations, Inclusions and Inequalities. Springer, Cham (2017)

5. Khodabakhshi, N., Vaezpour, S.M.: Existence and uniqueness of positive solution for a class of boundary value problems with fractional q-differences. J. Nonlinear Convex Anal. 16, 375-384 (2015)

6. Zhang, X., Liu, L., Zou, Y.: Fixed-point theorems for systems of operator equations and their applications to the fractional differential equations. J. Funct. Spaces 2018, 1-9 (2018)

7. Trigeassou, J.C., Maamri, N.: Initial conditions and initialization of linear fractional differential equations. Signal Process. 91(3), 427-436 (2011)

8. Ege, S.M., Topal, F.S.: Existence of positive solutions for fractional boundary value problems. J. Appl. Anal. Comput. 7(2), 702-712 (2017)

9. Wang, G., Pei, K., Chen, Y.: Stability analysis of nonlinear Hadamard fractional differential system. J. Franklin Inst. 356(12), 6538-6546 (2019). https://doi.org/10.1016/j.jfranklin.2018.12.033

10. Wang, G., Ren, X., Bai, Z., Hou, W.: Radial symmetry of standing waves for nonlinear fractional Hardy-Schrödinger equation. Appl. Math. Lett. 96, 131-137 (2019)

11. Jankowski, T: Boundary problems for fractional differential equations. Appl. Math. Lett. 28(2), 14-19 (2014)

12. Zhang, X., Liu, L., Wu, Y., Lu, Y.: The iterative solutions of nonlinear fractional differential equations. Appl. Math. Comput. 219, 4680-4691 (2013)

13. Zhang, L., Ahmad, B., Wang, G.: Successive iterations for positive extremal solutions of nonlinear fractional differential equations on a half-line. Bull. Aust. Math. Soc. 91, 116-128 (2015)

14. Cui, Y.: Uniqueness of solution for boundary value problems for fractional differential equations. Appl. Math. Lett. 51, 48-54 (2016)

15. Wei, Y., Song, Q., Bai, Z.: Existence and iterative method for some fourth order nonlinear boundary value problems. Appl. Math. Lett. 87, 101-107 (2019) 
16. Ding, Y., Wei, Z., Xu, J., et al.: Extremal solutions for nonlinear fractional boundary value problems with p-Laplacian. J. Comput. Appl. Math. 288, 151-158 (2015)

17. Zhang, X., Liu, L., $W u, Y$.: The uniqueness of positive solution for a fractional order model of turbulent flow in a porous medium. Appl. Math. Lett. 37, 26-33 (2014)

18. Chen, T., Liu, W., Hu, Z:: A boundary value problem for fractional differential equation with p-Laplacian operator at resonance. Nonlinear Anal. 75, 3210-3217 (2012)

19. Liu, X., Jia, M., Ge, W.: The method of lower and upper solutions for mixed fractional four-point boundary value problem with p-Laplacian operator. Appl. Math. Lett. 65, 56-62 (2017)

20. Tian, Y., Wei, Y., Sun, S.: Multiplicity for fractional differential equations with p-Laplacian. Bound. Value Probl. 2018, 127, $1-14(2018)$

21. Sheng, K., Zhang, W., Bai, Z: Positive solutions to fractional boundary value problems with p-Laplacian on time scales. Bound. Value Probl. 2018, 70 (2018)

22. Tian, Y., Sun, S., Bai, Z:: Positive solutions of fractional differential equations with p-Laplacian. J. Funct. Spaces 2017 $1-9(2017)$

23. Wang, T., Wang, G., Yang, X.: On a Hadamard-type fractional turbulent flow model with deviating arguments in a porous medium. Nonlinear Anal., Model. Control 22, 765-784 (2017)

24. Giampiero, P.: The Dirichlet problem for the p-fractional Laplace equation. Nonlinear Anal. 177, 699-732 (2018)

25. Li, A., Wei, C.: On fractional p-Laplacian problems with local conditions. Adv. Nonlinear Anal. 7, 485-496 (2018)

26. Yan, F., Zuo, M., Hao, X.: Positive solution for a fractional singular boundary value problem with p-Laplacian operator. Bound. Value Probl. 2018, 51, 1-10 (2018)

27. Goodrich, C.S.: Existence and uniqueness of solutions to a fractional difference equation with nonlocal conditions Comput. Math. Appl. 61(2), 191-202 (2011)

28. Zhang, L., Ahmad, B., Wang, G.: The existence of an extremal solution to a nonlinear system with the right-handed Riemann-Liouville fractional derivative. Appl. Math. Lett. 31(3), 1-6 (2014)

29. Liu, X., Jia, M.: Existence of solutions for the integral boundary value problems of fractional order impulsive differential equations. Math. Methods Appl. Sci. 39(3), 475-487 (2016)

30. Pei, K., Wang, G., Sun, Y.: Successive iterations and positive extremal solutions for a Hadamard type fractional integro-differential equations on infinite domain. Appl. Math. Comput. 312, 158-168 (2017)

31. Song, Q., Bai, Z:: Positive solutions of fractional differential equations involving the Riemann-Stieltjes integral boundary condition. Adv. Differ. Equ. 2018, 183, 1-7 (2018)

32. Wang, G., Pei, K., Agarwal, R., et al.: Nonlocal Hadamard fractional boundary value problem with Hadamard integral and discrete boundary conditions on a half-line. J. Comput. Appl. Math. 343, 230-239 (2018)

33. Zhang, S.: Monotone iterative method for initial value problem involving Riemann-Liouville fractional derivatives. Nonlinear Anal. 71, 2087-2093 (2009)

34. Wang, G.: Monotone iterative technique for boundary value problems of a nonlinear fractional differential equation with deviating arguments. J. Comput. Appl. Math. 236, 2425-2430 (2012)

35. Wang, G., Agarwal, R.P., Cabada, A.: Existence results and the monotone iterative technique for systems of nonlinear fractional differential equations. Appl. Math. Lett. 25, 1019-1024 (2012)

36. Wang, G., Baleanu, D., Zhang, L.: Monotone iterative method for a class of nonlinear fractional differential equations Fract. Calc. Appl. Anal. 15, 244-252 (2012)

37. Wang, G., Sudsutad, W., Zhang, L., Tariboon, J.: Monotone iterative technique for a nonlinear fractional q-difference equation of Caputo type. Adv. Differ. Equ. 2016, 211, 1-11 (2016)

38. Wang, G.: Explicit iteration and unbounded solutions for fractional integral boundary value problem on an infinite interval. Appl. Math. Lett. 47, 1-7 (2015)

39. Wang, G.: Twin iterative positive solutions of fractional q-difference Schrödinger equations. Appl. Math. Lett. 76, 103-109 (2018)

40. Zhai, C., Jing, R.: The unique solution for a fractional q-difference equation with three-point boundary conditions. Indag. Math. 29, 948-961 (2018)

41. Zhang, W., Bai, Z., Sun, S.: Extremal solutions for some periodic fractional differential equations. Adv. Differ. Equ. 2016 $179,1-8(2016)$

42. Zhang, L., Ahmad, B., Wang, G.: Existence and approximation of positive solutions for nonlinear fractional integro-differential boundary value problems on an unbounded domain. Appl. Comput. Math. 15, 149-158 (2016)

43. Bai, Z., Zhang, S., Sun, S., Yin, C.: Monotone iterative method for a class of fractional differential equations. Electron. J. Differ. Equ. 2016, 06, 1-8 (2016)

44. Jarad, F., Uǧurlu, E., Abdeljawad, T., Baleanu, D.: On a new class of fractional operators. Adv. Differ. Equ. 2017, 247, 1-16 (2017)

45. Abdeljawad, T:: On conformable fractional calculus. J. Comput. Appl. Math. 279, 57-66 (2015) 